\title{
Entrevista Motivacional no ensino de graduação em Odontologia
}

\author{
Nathalia Ribeiro Jorge da Silva*; Alexandre Emídio Ribeiro Silva**; Andreia Morales Cascaes** \\ * Mestranda, Programa de Pós-Graduação em \\ Odontologia, Universidade Federal de Pelotas \\ ** Doutor(a) em Epidemiologia, Professor(a), Faculdade \\ de Odontologia, Departamento de Odontologia Social \\ e Preventiva, Universidade Federal de Pelotas \\ Recebido em 13/09/2018. Aprovado em 11/07/2019.
}

\begin{abstract}
RESUMO
A Entrevista Motivacional (EM) é um método de aconselhamento que busca intervir no estilo de vida do indivíduo, com a finalidade de promover uma mudança comportamental benéfica à sua saúde. Embora bastante difundido em alguns países, o ensino da EM não é incorporado ao currículo da graduação das universidades brasileiras. O objetivo deste estudo é relatar a metodologia de desenvolvimento e a análise do desempenho de estudantes de graduação em Odontologia que participaram de um projeto piloto para o ensino teórico e prático da EM da Universidade Federal de Pelotas (UFPel). A etapa teórica foi conduzida por meio do Ambiente Virtual de Aprendizagem (Moodle UFPel) e, a prática foi desenvolvida junto a famílias de crianças cadastradas em uma Unidade Básica de Saúde, em Pelotas, RS, que oferece estágio curricular obrigatório para estudantes da Faculdade de Odontologia da UFPel. O desempenho dos estudantes era avaliado semanalmente por meio do instrumento Motivational Interviewing Treatment Integrity (MITI 3.1.1.). Os estudantes também preenchiam um questionário de autoavaliação. No geral, o projeto mostrou-se efetivo na melhoria do desempenho dos estudantes na aplicação da EM ao longo do estudo, com oscilações em algumas habilidades específicas. A EM é uma abordagem educativa inovadora e que demonstra resultados benéficos ao ensino da graduação em Odontologia.
\end{abstract}

Descritores: Entrevista Motivacional. Educação em Odontologia. Estudantes

\section{INTRODUÇÃO}

A Entrevista Motivacional (EM) é um método de aconselhamento que busca intervir no estilo de vida do indivíduo, com a finalidade de promover uma mudança comportamental benéfica a sua saúde ${ }^{1}$. Descrita inicialmente por Miller e
Rollnick (1983) no âmbito do alcoolismo, aos poucos foi demonstrando sua efetividade e sendo difundida mundialmente, aplicando-se a diversas áreas da saúde ${ }^{1}$.

Cientificamente comprovada na literatura em casos de transtornos alimentares, diabetes, HIV, 
saúde bucal, entre outros ${ }^{2}$, pesquisas demonstram seu efeito benéfico para a saúde ao orientar o paciente a encontrar suas próprias motivações para realizar as mudanças.

Estudos que avaliaram o ensino da EM em diversas áreas da saúde demonstraram maior confiança dos estudantes em lidar com as necessidades dos pacientes, além de aprimorar suas habilidades de comunicação interpessoal, resultando em mudanças benéficas, tais como maior adesão a tratamentos medicamentosos, mudanças positivas na dieta, aumento da prática de atividade física e perda de peso $^{2,3}$.

Embora os estudos realizados a nível mundial demonstrem a efetividade desse método na melhoria em condições de saúde gerais, no âmbito odontológico ainda há poucas evidências ${ }^{4}$. Apesar disso, os resultados são promissores, apontando para melhor autocuidado com a saúde bucal, com prevenção de doenças bucais como cárie, gengivite e placa dental ${ }^{2,5}$. Poucos estudos avaliaram o ensino e aprendizagem da EM no contexto da Odontologia. Resultados indicam que a abordagem auxiliou os estudantes na sua relação interpessoal com o paciente, a trabalhar com a ambivalência dos mesmos e, a orientá-los sobre mudanças quanto aos comportamentos em saúde bucal $^{5,8}$.

O ensino e a aprendizagem da EM por meio do treinamento de estudantes de diversas áreas de graduação em saúde possibilita o conhecimento desse método inovador, a ampliação de habilidades de manejo do paciente, além de aumentar a sua confiança para utilizá-lo $3,5,7,8$. A capacitação do futuro profissional para utilizar a mudança comportamental ao invés do reflexo de "consertar" o paciente, faz com que ele entenda e explore motivações, e coloque-se com empatia para fortalecer a autoestima, estimulando um resultado promissor ${ }^{1}$.

A estrutura curricular da Faculdade de Odontologia da Universidade Federal de Pelotas
(UFPel) contém apenas alguns conteúdos de educação em saúde e comunicação em poucas atividades, como nas disciplinas da "Unidade de Saúde Bucal Coletiva", onde é vivenciado o conhecimento da realidade da população usuária do Sistema Único de Saúde (SUS) e é estimulado o diálogo com a comunidade que frequenta a Unidade Básica de Saúde (UBS). Na disciplina de "Psicologia e Odontologia" trabalha-se a psique do ser humano, como entendê-lo e a colocar-se no seu lugar.

Apesar de algumas disciplinas abordarem o tema, necessita-se de uma maior abrangência dessa prática ao longo do curso, para que o futuro profissional saiba lidar com o outro e intervir de modo mais efetivo nos comportamentos de risco em saúde. Visando introduzir esse assunto no curso de graduação em Odontologia da UFPel, desenvolveu-se um projeto de ensino piloto teórico-prático para alicerçar os conceitos que compõem a EM e seus pré-requisitos para a execução do método direcionado à área odontológica. Este artigo relata a metodologia proposta e a análise do desempenho dos estudantes que participaram do projeto.

\section{METODOLOGIA}

$\mathrm{O}$ ensino e a aprendizagem da EM para estudantes de graduação em Odontologia da UFPel foi realizado por meio de um projeto de ensino piloto que os submeteu a treinamentos teóricos e práticos durante o período de julho a outubro de 2016. A população-alvo do projeto foram os estudantes que já haviam cursado a disciplina de "Unidade de Saúde Bucal Coletiva I" do $2^{\circ}$ ano e tinham a disponibilidade do turno da sexta-feira à tarde.

Os estudantes foram inicialmente capacitados por meio do Ambiente Virtual de Aprendizagem (AVA) da UFPel, utilizando a ferramenta Moodle como recurso didático. Conteúdos teóricos foram disponibilizados por 
meio de módulos que introduziam a EM, suas características e especificidades. Os estudantes executavam exercícios de reforço do material aprendido e obtinham um acompanhamento de seu aprendizado, com um feedback personalizado das tarefas entregues.

O módulo I introduziu a EM com seu histórico, conceitos, proposições e princípios norteadores. O módulo II focou na utilização da EM na área da saúde bucal e as evidências no âmbito da prática odontológica. O módulo III explanou o seu uso na realidade, contando com orientações de como executá-la nas atividades práticas do projeto. Por fim, no módulo IV foram inseridas as orientações finais para capacitar os estudantes a colocarem a EM em prática na comunidade.

A execução prática da EM foi realizada em parceria com uma UBS, pertencente ao estágio curricular obrigatório em Saúde Bucal Coletiva da Faculdade de Odontologia da UFPel. Um recorte da população de famílias com crianças foi realizado para a execução da parte prática do projeto. Os agentes comunitários de saúde acompanhavam os estudantes no deslocamento na área de abrangência da UBS, direcionando-os aos domicílios a serem visitados, assim como na apresentação do estudante à família. A seleção das famílias participantes ocorreu de forma aleatória, bastava o responsável legal e a criança estarem em casa e aceitarem o convite para participação.

As visitas duravam de 30 a 45 minutos. Ao iniciar a conversa, o estudante explicava o motivo da visita e seguia um roteiro estruturado que servia de guia para aplicação da EM. Perguntas abertas eram utilizadas para estimular o responsável a falar sobre os hábitos de saúde bucal, alimentares, valores relacionados à saúde geral e bucal e dificuldades e facilidades quanto ao uso e acesso aos serviços de saúde para a criança. Durante o desenvolvimento da conversa, as três habilidades básicas da EM (perguntar, escutar e informar) eram combinadas para identificar comportamentos relacionados com a saúde bucal desejáveis de mudança, ao mesmo explorar crenças, motivações e amplificar a discrepância do responsável pela criança em relação às mudanças. Um álbum ilustrado auxiliou no repasse de informações relevantes sobre prevenção de cárie na primeira infância, servindo de ferramenta para discutir um plano de mudança. Ao final, um resumo da conversa era realizado pelo estudante, por meio de escuta reflexiva, ressaltando os principais aspectos discutidos na visita. Nesse dia, a criança era agendada para uma consulta de rotina com o cirurgião dentista da UBS.

O diálogo da visita era gravado, com a autorização do participante. O estudante incluía o áudio no Dropbox e na semana seguinte recebia feedback a respeito do seu desempenho, baseado no Motivational Interviewing Treatment Integrity (MITI 3.1.1.) $)^{6}$, que é o "Instrumento de Avaliação da Fidelidade da Entrevista Motivacional”. O MITI 3.1.1. é um sistema de codificação comportamental utilizado para facilitar a obtenção do feedback da EM, estabelecendo parâmetros para iniciantes e experientes. As audições foram realizadas e avaliadas pela bolsista de ensino do projeto, com experiência teórica e prática prévia em EM.

O MITI 3.1.1 fornece feedback personalizado, visto que avalia os estudantes segundo os princípios norteadores da EM de motivação, colaboração, respeito a autonomia, orientação para a mudança e empatia, além de determinar um escore global do desempenho da entrevista. $\mathrm{O}$ escore global é obtido pela média da soma das notas de 1 a 5 nos princípios norteadores, podendo atingir a nota máxima de 5 , classificando como nível iniciante aqueles indivíduos que alcançarem a pontuação de 3,5 a 3,9 e como experientes aqueles com pontuação mínima de 4,0. O MITI 3.1.1. avalia concomitantemente as habilidades básicas da EM para informar, perguntar e escutar. Nesses itens avalia-se a 
proporção de perguntas abertas em relação às fechadas, o quanto de reflexões simples em oposição às complexas são obtidas e o quanto de informações aderentes são repassadas, ou seja, aquelas sem confrontar o paciente. Observando tudo isso se obtém uma análise do perfil do entrevistador e da entrevista.

As habilidades básicas também possuem parâmetros para iniciantes e experientes, sendo eles: para o quesito de perguntas abertas de $50 \%$ a $69 \%$ para iniciantes e mínimo de $70 \%$ para experientes. As reflexões complexas variam de $40 \%$ a $49 \%$ para iniciantes e mínimo de $50 \%$ para experientes e, o repasse de informações aderentes de $90 \%$ a $99 \%$ para iniciantes e mínimo de $100 \%$ para experientes.

O estudante a cada entrevista recebia a avaliação do seu desempenho, e era orientado a enfrentar suas dificuldades na prática, com vistas ao aprimoramento da EM.

Além do feedback do MITI 3.1.1., o estudante realizava ao final da entrevista a sua autoavaliação e o seu grau de percepção sobre o interesse e a motivação do entrevistado. Essa informação era coletada quando o estudante preenchia um checklist da sua visita no domicílio e respondia às perguntas: "Como você avalia a abordagem realizada por você?" e "Como você avalia o grau de interesse/motivação do participante no que foi discutido na visita?". As opções de resposta variavam de 0 (muito ruim), 1 (ruim), 2 (regular), 3 (boa) a 4 (muito boa).

Os dados foram coletados durante a realização das EM e digitados em planilhas no Microsoft Excel versão 2010. Foram realizadas análises descritivas dos resultados. O estudo foi aprovado pelo Comitê de Ética e Pesquisa da Faculdade de Odontologia da UFPel sob parecer número 2.517.478.

\section{RESULTADOS}

O presente estudo avaliou 27 Entrevistas Motivacionais realizadas pelos três acadêmicos participantes do Projeto de Ensino.

\section{Desempenho geral dos estudantes}

A tabela 1 descreve os resultados gerais para a equipe de estudantes. A análise da equipe como um todo demonstrou que o desempenho quanto ao escore global, que caracteriza toda a interação da entrevista, variou de uma média de 3,9 inicialmente até atingir 4,2 no final, ou seja, desde o início já atingiram o escore de iniciantes de 3,5 e ao final superaram o nível de experiente de 4,0. Nesse quesito, os estudantes evoluíram durante a execução do projeto.

Tabela 1. Evolução dos Acadêmicos da Faculdade de Odontologia da UFPel durante o projeto sobre a utilização da entrevista motivacional

\begin{tabular}{lccc}
\hline Critérios de avaliação MITI 3.1.1. & Início & Meio & Final \\
\hline Escore global (média) & 3,9 & 4,0 & 4,2 \\
Evocação (média) & 3,5 & 3,7 & 4,3 \\
Colaboração (média) & 4,1 & 4,0 & 4,4 \\
Respeito a autonomia (média) & 4,3 & 4,1 & 4,2 \\
Orientação para mudança (média) & 3,8 & 3,8 & 4,0 \\
Empatia (média) & 3,8 & 4,4 & 4,1 \\
Perguntas abertas (\%) & 50,9 & 37,0 & 46,9 \\
Reflexões complexas (\%) & 28,6 & 37,3 & 29,6 \\
Informação aderente (\%) & 90,4 & 83,5 & 87,6 \\
Autoavaliação (média) & 2,4 & 3,0 & 3,3 \\
Percepção sobre a entrevista (média) & 3,5 & 3,3 & 3,7 \\
\hline
\end{tabular}


$\mathrm{O}$ número de perguntas abertas analisa o comportamento do entrevistador em permitir uma ampla quantidade de possíveis respostas para detecção de hábitos deletérios. Os acadêmicos obtiveram uma redução de 50,9\% para $46,9 \%$ nesta proporção, sendo que o esperado para iniciantes é de $50 \%$. A frequência de reflexões complexas do MITI 3.1.1. é de $40 \%$ para iniciantes e, para experientes $50 \%$. Na equipe houve um crescimento de $28,6 \%$ para $29,6 \%$, passando por um período intermediário de $37,3 \%$. A respeito do repasse de informações aderentes obtido pela equipe, constata-se que esteve entre $90,4 \%$ a $87,6 \%$, sendo que o parâmetro de iniciante é de $90 \%$, e que foi alcançado em alguns momentos.

A autoavaliação a respeito da abordagem realizada durante a entrevista era ranqueada pelo próprio estudante, assim como a percepção sobre a entrevista de acordo com o grau de interesse e motivação do entrevistado. Os estudantes passaram de uma autoavaliação "regular" para uma "boa" e o nível da percepção do interesse/motivação do entrevistado ficou como "boa".

\section{Desempenho individual dos estudantes}

A tabela 2 apresenta os resultados individuais dos estudantes. $\mathrm{O}$ estudante 1 evoluiu nos quesitos de escore global, alcançando em vários momentos o escore de experiente $(4,0)$. Na quantidade de perguntas abertas variou de $57,6 \%$ a $38,8 \%$, alcançando os $50 \%$ de iniciante em partes das entrevistas, entretanto finalizou com $45,7 \%$. As reflexões complexas foram evoluindo do início para o meio do processo, porém no final houve uma diminuição e chegou ao percentual de $0 \%$. A informação aderente variou de $97,3 \%$ a $88,6 \%$, atingido os $90 \%$ de iniciante, finalizando com 92,3\%. A autoavaliação sobre a entrevista passou do nível "ruim" para o "regular" e a percepção sobre a entrevista demonstrou que o interesse dos entrevistados foi bom, sendo observado melhora nas entrevistas intermediárias e finais.

Tabela 2. Evolução dos Acadêmicos da Faculdade de Odontologia da UFPel sobre a utilização da entrevista motivacional

\begin{tabular}{lccc|ccc|ccc}
\hline \multirow{2}{*}{ Evolução } & \multicolumn{3}{c}{ Estudante 1 } & \multicolumn{3}{c}{ Estudante 2 } & \multicolumn{3}{c}{ Estudante 3 } \\
\cline { 2 - 10 } & Início & Meio & Final & Início & Meio & Final & Início & Meio & Final \\
\hline Escore global (média) & 3,8 & 4,1 & 3,9 & 4,2 & 4,1 & 4,5 & 3,8 & 4,0 & 4,2 \\
Evocação (média) & 3,3 & 4,0 & 4,0 & 4,0 & 3,6 & 4,6 & 3,3 & 3,6 & 4,3 \\
Colaboração (média) & 4,0 & 4,3 & 4,0 & 4,3 & 4,3 & 4,3 & 4,0 & 3,6 & 5,0 \\
Respeito a autonomia (média) & 4,0 & 3,6 & 4,0 & 4,3 & 4,3 & 4,6 & 4,6 & 4,6 & 4,0 \\
Orientação para mudança (média) & 3,6 & 4,0 & 3,6 & 4,3 & 3,6 & 4,6 & 3,6 & 4,0 & 4,0 \\
Empatia (média) & 4,0 & 4,6 & 4,0 & 4,3 & 4,6 & 4,3 & 3,3 & 4,0 & 4,0 \\
Perguntas abertas (\%) & 57,6 & 38,8 & 45,7 & 55,6 & 36,3 & 52,7 & 39,7 & 36,1 & 42,3 \\
Reflexões complexas (\%) & 24,3 & 38,1 & - & 43,3 & 44,5 & 48,3 & 28,3 & 30,6 & 41,6 \\
Informação aderente (\%) & 97,3 & 88,6 & 92,3 & 80,7 & 89,6 & 80,6 & 93,2 & 72,4 & 89,9 \\
Autoavaliação (média) & 1,6 & 2,6 & 2,6 & 3,0 & 3,6 & 4,0 & 2,6 & 3,0 & 3,3 \\
Percepção sobre a entrevista (média) & 3,0 & 3,6 & 3,6 & 4,0 & 3,3 & 4,0 & 3,6 & 3,0 & 3,6 \\
\hline
\end{tabular}

O estudante 2 já começou as entrevistas com escore de experiente, ou seja, as entrevistas avaliadas obtiveram escores maiores que 4,0. Quanto as perguntas abertas ele começou como nível iniciante com 55,6\%, passou para 36,3\% e finalizou com $52,7 \%$, alcançando a pontuação final de iniciante. As reflexões complexas evoluíram de $43,3 \%$ para $48,3 \%$, atingindo o nível iniciante de 
40\%. A informação aderente passou por um crescimento do início para o meio das entrevistas, de $80,7 \%$ para $89,6 \%$, sendo que para iniciante é $90 \%$, entretanto finalizou com 80,6\%. A autoavaliação foi avaliada de "boa" inicialmente e encerrou como "muito boa", assim como o grau de interesse dos entrevistados que em grande parte foi "muito bom".

O estudante 3 começou com escore global de iniciante $\mathrm{e}$ finalizou com escore experiente, variando de 3,8 para 4,2. A sua quantidade de perguntas abertas evoluiu de $39,7 \%$ para $42,3 \%$, não alcançando o grau de iniciante. As reflexões complexas obtiveram uma significativa melhoria, passando de $28,3 \%$ para $41,6 \%$, alcançando o nível de iniciante de 40\%. A informação aderente alcançou em alguns momentos o escore de iniciante de $90 \%$, com 93,2\%, entretanto oscilou para $72,4 \%$ e finalizou com $89,9 \%$. A autoavaliação do estudante passou de "regular" para "boa" e a percepção da entrevista se manteve constante no nível "bom".

\section{DISCUSSÃO}

A capacitação dos estudantes de Odontologia da UFPel em EM mostrou-se efetiva considerando que o critério de escore global aumentou das entrevistas iniciais para as finais e, é ela que nos informa a impressão geral do perfil do entrevistador, alcançando assim o escore de experiente.

Algumas oscilações no desempenho de perguntas abertas, informações aderentes e reflexões complexas foram observadas ao longo do estudo, constatando-se a importância do reforço contínuo das habilidades básicas da abordagem. Por outro lado, a autoavaliação do estudante evoluiu com o passar das entrevistas, demonstrando uma confiança para aplicar a EM, assim como também houve evolução a respeito da percepção sobre o interesse do entrevistado.

A qualidade geral das evidências dos estudos utilizando a EM apontam a relevância do uso deste método inovador para a capacitação dos futuros profissionais. Os estudos publicados têm descrito o treinamento da EM por meio de diversas estratégias pedagógicas. Na área da farmácia, os estudantes de $3^{\circ}$ e $4^{\circ}$ anos da Universidade de Missouri (EUA) foram treinados para realizarem a EM e, se reuniam uma vez na semana durante três horas por quatro meses. Eles possuíam supervisão individual à medida que aprendiam as habilidades da EM e contavam com coorientadores de doutorado. A logística do curso era através da realização de leituras obrigatórias, palestras interativas, demonstração através de vídeos, uma prática em grupo ou individual, além da supervisão individualizada e do preenchimento de formulários de reflexão. A parte prática da EM contava com um questionário para promover interação durante uma conversa com o paciente que durava de cinco a 20 minutos. A conversa era gravada e após, faziam um resumo escrito. A avaliação ocorria através do Health Care Climate que é de domínio público para avalição da percepção dos pacientes sobre as habilidades de aconselhamento dos estudantes. Os supervisores forneciam um feedback e orientações para os estudantes após ouvirem a gravação e lerem os formulários de reflexão e, os classificavam conforme a abordagem. Além disso, estimulavam a confiança para melhoria na aplicação do método, resultando em alunos com as habilidades necessárias para sua execução e altamente motivados para colocá-la em prática após o término do curso $^{7}$. O presente estudo assim como o descrito, apresentava a EM sob diversas estratégias pedagógicas para melhor exemplificar aos acadêmicos sobre o método. $\mathrm{O}$ uso de questionário estruturado para estimular o diálogo, a gravação da entrevista e o feedback repassado aos entrevistadores foram pontos em comum encontrados.

Estudantes do $2^{\circ}$ ano de Higiene Dental da Faculdade Comunitária de Midwestern (EUA) 
foram convidados a participar de um estudo piloto de EM. O dialogo dos estudantes com os pacientes foram gravados durante duas sessões. No primeiro momento, a gravação era realizada sem o estudante ter conhecimento sobre as técnicas da EM, após recebiam o feedback e aprendiam a EM. O conteúdo do treinamento eram leituras obrigatórias, duas sessões, uma de quatro horas e outra de três horas de duração, em que os alunos discutiam sobre o assunto, praticavam exercícios e aprendiam algumas formas de comunicação. A avaliação individual era realizada através do MITI 3.1.1., tal qual no presente estudo ${ }^{8}$. Os estudantes melhoram seus escores comparando a entrevista antes do treinamento da EM e a posterior já tendo tal conhecimento, entretanto não houve melhora na relação de reflexões e perguntas ${ }^{8}$. A logística entre o estudo realizado e o presente foi diferente ao testar o acadêmico antes e depois do conhecimento da EM, mas o método avaliativo foi semelhante ao fazer uso do MITI 3.1.1. para fornecimento do feedback individualizado de cada acadêmico e de cada entrevista.

A capacitação dos estudantes de Odontologia por meio deste projeto piloto, mesmo que com uma amostra de apenas três estudantes permitiu identificar aonde devemos focar para melhorar o aprendizado, visto que vários escores tiveram oscilações durante o estudo. As habilidades básicas de perguntar, escutar e informar devem ser aprimoradas e reforçadas aos estudantes continuamente, talvez com mais treinamento teórico e principalmente prático. Dessa forma, a probabilidade de retornar a abordagem educativa tradicional, corriqueira no contexto da Faculdade de Odontologia da UFPel, pode ser reduzida. Os estudantes que participaram deste projeto de ensino também realizavam outras atividades de contato com o paciente no interior da faculdade. Logo, alguma confusão para aplicar o método poderia acontecer pela duplicidade de abordagens, uma possível explicação para as oscilações que aconteceram ao longo do estudo.

A diferença de aprendizado entre os alunos foi observada, os estudantes 2 e 3 tiveram um desempenho mais regular durante o projeto, até melhoraram em alguns aspectos, já o aluno 1 aparentemente apresentou maior dificuldade na execução do método. Um possível motivo é o fato do estudante 1 ser mais inexperiente no quesito de contato com o paciente, pois estava apenas começando sua atuação na clínica. Os estudantes 2 e 3 eram de semestres mais avançados e, já possuíam esse contato, gerando uma maior experiência de comunicação interpessoal com o paciente.

Os métodos utilizados pelo projeto (AVA, Dropbox e a discussão presencial com os alunos), podem ser aprimorados com discussões presenciais e carga horária prática, justamente para aumentar a confiança e reforçar a importância de cada uma das habilidades e princípios norteadores da EM. Segundo Bray et al. (2013) ${ }^{5}$ a abordagem integrada de treinamento didático e prático é o melhor método de ensino na EM. Em seu estudo, os autores utilizaram aulas teóricas e a aplicação durante a clínica curricular da Faculdade de Higiene Dental para implementar a EM e, que resultou num impacto positivo de desempenho dos acadêmicos em habilidades da $\mathrm{EM}^{5}$.

Por tratar-se de um projeto piloto, é possível analisar os resultados e aprimorar as estratégias pedagógicas para melhoria na qualidade do ensino e aprendizagem dos estudantes de Odontologia, principalmente tendo como alicerce a literatura que relata o quanto a EM é efetiva e demonstra resultados positivos na intervenção em problemas de saúde.

\section{CONCLUSÕES}

Considerando o fato de os estudantes não possuírem conhecimento prévio do método, eles conseguiram desenvolver as habilidades básicas da EM, mesmo que não alcançando em sua totalidade 
os parâmetros estabelecidos pelo MITI 3.1.1. Tal fato também nos faz refletir sobre itens que podem ser reforçados para uma melhor obtenção dos escores, como por exemplo a habilidade de fazer perguntas abertas e evitar de se fazer as perguntas fechadas, para então se obter um dado mais completo a respeito da realidade do paciente. Quanto as reflexões, deve-se estimular o parar e pensar sobre o que é relatado, fazer uma escuta reflexiva e qualitativa, para posteriormente ser capaz de fazer uma reflexão a respeito.

O desenvolvimento do projeto com auxílio do AVA facilitou o aprendizado, uma vez que essa ferramenta é disponibilizada pela UFPel e utilizada em algumas disciplinas de graduação. O MITI 3.1.1. foi o método avaliativo de escolha dado sua praticidade em fornecer o feedback, além de ser um método recomendado previamente na literatura.

A inserção do projeto foi realizada em uma etapa adequada do curso, visto que no $2^{\circ}$ e $3^{\circ}$ anos se inicia o contato com os pacientes. Com o passar das entrevistas, os estudantes desenvolveram mais confiança e melhoraram sua relação interpessoal. A EM é uma abordagem educativa inovadora e que demonstra resultados benéficos ao ensino da graduação em Odontologia.

\section{ABSTRACT \\ Motivational Interview training for dental students}

Motivational Interviewing (MI) is a counseling method that seeks to intervene in an individual's lifestyle in order to promote behavioral change that is beneficial to their health. Although widespread in some countries, MI training is not incorporated into the curriculum of Brazilian universities. The objective of this study is to report on the development of a pilot project for MI training at the Federal University of Pelotas (UFPel), and the performance of dental students who participated in this project. The theoretical phase was conducted through a Virtual Learning Environment (Moodle UFPel) and the practical phase was developed with families of children enrolled at a Primary Health Care Center in
Pelotas, RS, which offers internship for dental students. Student performance was assessed weekly using the Motivational Interviewing Treatment Integrity instrument (MITI 3.1.1.). Students also completed a self-assessment questionnaire. Overall, the project was effective in improving students' performance in applying MI over the study period, with variations in some specific skills. MI is an innovative educational approach that demonstrates beneficial results for dental students.

Descriptors: Motivational Interview. Dental Education. Students.

\section{AGRADECIMENTO}

O presente trabalho foi realizado com apoio da Coordenação de Aperfeiçoamento de Pessoal de Nível Superior - Brasil (CAPES) - Código de Financiamento 001

\section{REFERÊNCIAS}

1. Rollnick S, Miller WR, Butler CC. Entrevista motivacional no cuidado da saúde: ajudando pacientes a mudar o comportamento. Porto Alegre: Artmed, 2009.

2. Martins RK, Mcneil DW. Review of Motivational Interviewing in promoting health behaviors. Clin Psy Rev. 2009; 29: 283-93.

3. Gecht-silver M, Lee D, Ehrlich-jones L, Bristow M. Evaluation of motivational interviewing training for third-year medical students. Fam Med. 2016; 48(2): 132-5.

4. Cascaes AM, Bielemann RM, Clark VL. Effectiveness of motivational interviewing at improving oral health: a systematic review. Rev Saúde Púb. 2014; 48: 142-53.

5. Bray KK, Catley D, Voelker MA, Liston R, Williams KB. Motivational interviewing in dental hygiene education: Curriculum modification and evaluation. J Dent Educ. 2013; 77(12): 1662-69.

6. Moyers TB, Martin T, Manuel JK, Miller 
WR, Ernst D. Revised Global Scales: Motivational Interviewing Treatment Integrity 3.1.1 (MITI 3.1.1) [Draft Manuscript]. Albuquerque (CASAA): University of New Mexico; 2010.

7. Goggin K, Hawes SM, Duval ER, Spresser CD, Martínez DA, Lynam I, Barnes A, Hinton-dampf AM, Murphy ME, Marken PA, Catley D. A motivational interviewing course for pharmacy students. Amer J Pharm Educ. 2010; 74(4 Suppl 70).
8. Croffoot C, Bray KK, Black MA, Koerber A. Evaluating the effects of coaching to improve Motivational Interviewing skills of dental hygiene students. J of Dental Hyg. 2010; 84(2): 57-64.

\section{Correspondência para:}

Andreia Morales Cascaes

e-mail: andreia.cascaes@ufpel.edu.br andreiacascaes@gmail.com

Faculdade de Odontologia

Departamento de Odontologia Social e Preventiva Universidade Federal de Pelotas

Rua Gonçalves Chaves, 457, sala 402b 96015-560 Pelotas/RS 